\title{
Flow cytometric characterization of cell surface markers to differentiate between fibroblasts and mesenchymal stem cells of different origin
}

\author{
Suzanne A. Sober ${ }^{1}$, Homa Darmani ${ }^{1}$, Dana Alhattab ${ }^{2}$, Abdalla Awidi ${ }^{3}$
}

\author{
${ }^{1}$ Jordan University of Science and Technology, Jordan \\ ${ }^{2}$ King Abdullah University of Science and Technology, Saudi Arabia \\ ${ }^{3}$ University of Jordan, Jordan
}

Submitted: 31 May 2020; Accepted: 2 December 2020

Online publication: 5 April 2021

Arch Med Sci

https://doi.org/10.5114/aoms/131088

Copyright ( 2022 Termedia \& Banach

\begin{abstract}
Introduction: Identification and purification of mesenchymal stem cells (MSCs) expanded in culture for therapeutic use is crucial for improved yield and optimal results. Fibroblasts are the most common cell type in connective tissue and are commonly found as contaminants of MSC cultures, affecting cell yield and potentially causing tumour formation after cell transplantation. In the current study, we wished to identify cell surface markers that can differentiate MSCs of different origins from fibroblasts.

Material and methods: Mesenchymal stem cells were isolated from bone marrow, adipose tissue, Wharton's jelly, and placental tissue, and fibroblasts were isolated from foreskin (as a negative control) in order to examine the differences in the expression of a panel of 14 different cell surface markers using multiplex flow cytometry.

Results: Our results indicate that the following markers could be useful in differentiating between fibroblasts and MSCs derived from the following: adipose tissue - CD79a, CD105, CD106, CD146, and CD271; Wharton's jelly - CD14, CD56, and CD105; bone marrow - CD105, CD106, and CD146; and placental tissue - CD14, CD105, and CD146. Furthermore, we found that, contradictory to previous studies, CD26 is not fibroblast specific.

Conclusions: The results of our study indicate that cell surface markers may prove to be a useful tool in the discrimination between MSCs of different origins and fibroblasts, and thus may be used to authenticate the identity of the isolated cells.
\end{abstract}

Key words: mesenchymal stem cells, adipose tissue, placenta, Wharton jelly, bone marrow.

\section{Introduction}

Human mesenchymal stem cells (MSCs) are found in almost all dividing tissues. They function in the renewal of dying cells within the tissues by differentiation of cells generated from stem cells [1]. This function has made them a target of interest for clinicians and researchers for many years, in the hope that they may be exploited for the treatment of various diseases in which tissues are damaged or have impaired function. Stem cell therapy has been useful in treating various diseases including multiple sclerosis [2], diabetic foot ulcers [3], cancer [4], arthritis [5],

\author{
Corresponding author: \\ Prof. Homa Darmani \\ Jordan University of Science \\ and Technology \\ Jordan \\ E-mail: darmani@just.edu.jo
}


spinal cord injuries [6], acute lung injuries [7], ischaemic heart disease [8], and cerebral hypoxic ischaemia [9].

Mesenchymal stem cells have been isolated from several tissues including bone marrow, adipose tissue, Wharton's jelly and placental tissue, periosteum, synovial membrane, dermis, muscle, and dental pulp [10]. However, MSCs make up a very small percentage of the cell population found within these tissues. In bone marrow, for example, MSCs comprise 0.01 to $0.001 \%$ of human bone marrow mononuclear cells [11]. This has presented a challenge to their isolation from various tissues, which in turn has led to the need for methods to authenticate the identity of the isolated cells.

Mesenchymal stem cells are identified in several ways. Apart from their spindle-like morphology in culture and their ability to attach to plastic surfaces, they can also differentiate into adipocytes, osteocytes and chondrocytes. Furthermore, the International Society for Cellular Therapy (ISCT) proposed that MSCs should express CD105, CD73, and CD90 and lack expression of CD45, CD34, CD14 or CD11b, CD79alpha or CD19, and HLA-DR surface molecules [12, 13]. However, there are data indicating the expression of some of these positive markers on a variety of other cell types [14]. Furthermore, CD34, which was declared by the ISCT as a negative surface marker in MSCs, has been shown to be expressed in native MSCs, like adipose tissue [15]. It is therefore logical to say that there is still a degree of disagreement regarding both the expression of markers and their percentage expression in MSCs from different sources and from different passage numbers.

Fibroblasts are the most common cell type in connective tissue and commonly contaminate MSC cultures. This can be an issue in the case of MSC culturing for clinical purposes, because transferring MSCs to patients from cultures that have been contaminated with fibroblasts could lead to tumour formation [16]. Fibroblasts have characteristics similar to those of MSCs in that they have similar morphology in culture, possess immune modulatory properties, and are capable of differentiating into adipocytes, osteocytes, and chondrocytes [17]. They also adhere to plastic and have similar surface marker expression.

So far, no markers have been established to discriminate between MSCs and fibroblasts [16]. One study concluded that the expression of CD166 was significantly higher and that of CD9 was significantly lower in MSCs than in fibroblasts. It also suggested that CD146 expression occurred in MSCs and not in fibroblasts, whereas CD106 expression in MSCs was at least tenfold higher than in fibroblasts [16]. In another study, CD106, CD146, and ITGA11 expression was MSC specific, while the expression of CD10 and CD26 was fibroblast specific [18]. Furthermore, CD44, CD90, and CD105 were also found to be non-specific for MSCs; pure human embryonic fibroblasts were also found to be positive for these markers [18]. Watson et al. [19] concluded that CD271 is the most specific marker for bone marrow-derived MSCs.

It is therefore clear that there has been no consensus as to which markers are specific for MSCs and which are specific for fibroblasts, especially with varying degrees of expression at different passages for both cell types. Thus, the main aim of the present study was in helping to establish cell surface markers that will enable definitive discrimination between MSCs and fibroblasts in order to authenticate cell lines isolated from various tissues, primarily in the hope of preventing the complications that may arise when MSCs are cultured for clinical applications.

\section{Material and methods}

\section{Material}

All tissue culture reagents and buffers were purchased from Gibco Laboratories, USA. Innohep was purchased from LEO Pharma, Denmark. All the fluorophore-conjugated monoclonal antibodies were purchased from BD Biosciences, USA, except for CD106 which was purchased from Invitrogen, Canada.

\section{Cell isolation and culture}

Only samples from patients with research consent were included. All procedures followed for the obtaining of tissue samples were in accordance with the Institutional Review Board Committee on human experimentation (King Abdullah University Hospital, Jordan University of Science and Technology). Cells were cultured routinely using $\alpha$-MEM (supplemented with $5 \%$ platelet lysate [prepared from pooled platelets] [20]), 1\% Penicillin/Streptomycin [P/S], $3 \mathrm{IU} / \mathrm{ml}$ Innohep and $2 \mathrm{mM}$ L-Glutamine) or DMEM (supplemented as above but without L-Glutamine).

\section{Fibroblast isolation from foreskin}

Three foreskin samples were collected from newborns at Al Amal Maternity Hospital (Amman, Jordan) after receiving maternal consent. The samples were washed (PBS containing 3\% P/S), cut into long, thin pieces and exposed to Dispase II (2.4 units/ml) for 16 hours at $40^{\circ} \mathrm{C}$. The epidermis was then peeled off the dermis and discarded, and the dermis was washed and exposed to 
collagenase $(0.35 \%)$ at $37^{\circ} \mathrm{C}$ for 60 minutes with constant shaking. The solution was then centrifuged at $400 \mathrm{~g}$ for 7 minutes, and the disintegrated dermis was removed using a cell strainer. The isolated cells were cultured in DMEM at $37^{\circ} \mathrm{C}$ in a humidified atmosphere of $5 \% \mathrm{CO}_{2}$ until $\leq 80 \%$ confluent.

\section{Adipose tissue-derived mesenchymal stem cells}

Three adipose tissue samples were obtained from liposuction procedures performed on patients at Jordan University Hospital (Amman, Jordan). Equal volumes of sample and $75 \%$ collagenase solutions were combined and incubated at $37^{\circ} \mathrm{C}$ for 30 minutes with continuous shaking. The medium was then added and the samples were centrifuged at $1200 \mathrm{~g}$ for 10 minutes, and the remaining solid portion of the adipose tissue was discarded. The pellet stromal vascular fraction was washed with red blood cell lysate until red blood cells were no longer visible. Isolated cells were then cultured in $\alpha-M E M$ at $37^{\circ} \mathrm{C}$ in a humidified atmosphere of $5 \% \mathrm{CO}_{2}$ until reaching $\leq 80 \%$ confluence.

\section{Bone marrow-derived mesenchymal stem cells}

Bone marrow aspirates were taken from the iliac crest of adult donors; nucleated cells were isolated using a density gradient (Ficol-Paque; Amersham Pharmacia Biotech, USA). The isolated cells were then cultured in $\alpha-M E M$ at $37^{\circ} \mathrm{C}$ in a humidified atmosphere of $5 \% \mathrm{CO}_{2}$ until reaching $\leq$ $80 \%$ confluence.

\section{Wharton's jelly-derived mesenchymal stem cells}

Mesenchymal stem cells from Wharton's jelly were provided by the Cell Therapy Centre at the University of Jordan, Amman, Jordan. The cells were isolated from umbilical cords (UCs) collected from donors at Jordan University Hospital following caesarean section, in order to minimize microbial contamination. The UCs were rinsed with PBS containing 3\% $\mathrm{P} / \mathrm{S}$ and cut into 5 -cm-long pieces. Following another rinsing with PBS containing 3\% $\mathrm{P} / \mathrm{S}$, they were cut longitudinally to expose the arteries and vein, which were removed. The remaining tissue was rinsed with PBS containing $3 \% \mathrm{P} / \mathrm{S}$ and cut into small pieces $\left(4 \mathrm{~mm}^{2}\right)$ and dispensed into tissue culture plates. These explants were allowed to attach for 10 minutes, and then $\alpha$-MEM was added gently to the plates and the cells were incubated in a humidified atmosphere of $5 \% \mathrm{CO}_{2}$ at a temperature of $37^{\circ} \mathrm{C}$ for $7-8$ days. Following this, the medium was replaced with fresh medi- um every 3 days until individual colonies reached $\leq 80 \%$ confluence.

\section{Placenta-derived mesenchymal stem cells}

Placenta-derived MSCs were isolated from 3 placentas collected by consent of the mother. The chorionic villi from the foetal portion were cut into pieces, washed, and incubated with TrypLE Select Enzyme (1X) diluted in PBS containing DNase (271 unit/ml) and $\mathrm{P} / \mathrm{S}$ with gentle rotation at $4^{\circ} \mathrm{C}$ overnight. The cells were then washed 3 times with PBS and then seeded into 6-well plates for $30-40$ minutes at $37^{\circ} \mathrm{C}$ in a humidified atmosphere of $5 \% \mathrm{CO}_{2}$ to allow the cells to adhere. After this, $2 \mathrm{ml}$ of $\alpha-M E M$ was added to each well, and the plates were incubated at $37^{\circ} \mathrm{C}$ until the cells reached subconfluency $(\leq 80 \%)$.

\section{Flow cytometry}

For all cell types, subconfluent cells ( $\leq 80 \%)$ were used after reaching Passage 3 . Cells were harvested using $0.25 \%$ trypsin and then washed using PBS containing $1 \%$ P/S and fluorophore-conjugated monoclonal antibodies were added in the combinations displayed in Table I, using the standard recommended quantities recommended by the manufacturer of each fluorophore-conjugated antibody. They were then incubated for 20 minutes in the dark. This was followed by centrifugation at $350 \mathrm{~g}$ for 5 minutes and cells were resuspended in PBS, after which the cells were analysed by flow cytometry and data on the cell surface markers was collected. Isotype controls were run parallel to test samples. Triplicate samples were analysed for each cell type.

\section{Statistical analysis}

Percentage fluorescence data of each sample were extracted from the dot plots obtained using flow cytometric analysis. One-way ANOVA was then performed to determine the presence of significant variation in expression of the surface markers $(p<0.05)$. Follow-up analysis using Tukey's pairwise comparison was then performed to determine the exact source of significant variation in surface marker expression.

\section{Results}

The expression of the surface markers CD26, CD34, CD44, CD73, CD90, and CD166, and HLA$A B C$ were very similar in stem cells derived from Wharton's jelly, adipose tissue, bone marrow, and placental tissue and fibroblasts derived from foreskin (ANOVA P>0.05) (Figure 1). However, significant differences were found in the expression of CD14, CD56, CD79a, CD105, CD106, CD146, and 


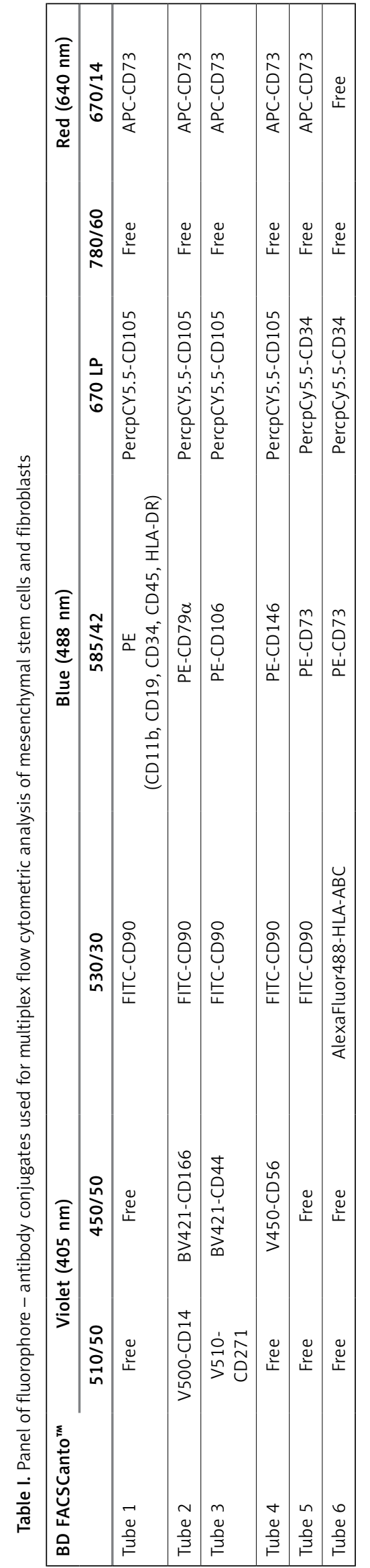

CD271 among these cells types (ANOVA P $<0.05$ )

(Figure 2).

\section{CD14}

Figure 3 shows the percentage of CD14-positive stem cells derived from Wharton's jelly, adipose tissue, bone marrow, placental tissue, and fibroblasts derived from foreskin. One-way ANO$\checkmark A$ revealed significant differences in expression of CD14 between these cells $(p<0.05)$. Follow-up analysis using Tukey's pairwise comparison revealed that the expression of CD14 in stem cells derived from Wharton's jelly (3\%) and placental tissue (4\%) was significantly lower than that observed in fibroblasts from foreskin (92\%) and stem cells derived from bone marrow $(85 \%)$ and adipose tissue (97\%).

\section{CD56}

The percentage of CD56-positive stem cells is shown in Figure 4. Significant differences in the levels of expression of CD56 were observed in the different cell types $(p<0.05$; ANOVA). Tukey's pairwise comparison confirmed that expression of CD56 was significantly higher in stem cells derived from Wharton's jelly (32\%) than in fibroblasts $(0.3 \%)$ and stem cells derived from adipose tissue $(0.4 \%)$, bone marrow (4\%), and placental tissue (4\%).

\section{CD79a}

The percentage of CD79a-positive stem cells derived from Wharton's jelly, adipose tissue, bone marrow, placental tissue, and fibroblasts derived from foreskin is shown in Figure 5. Significant differences in the percentage of CD79a-positive stems cells were observed these cells $(p<0.05$; ANOVA). Tukey's pairwise comparison revealed that the percentage of CD79a-positive stem cells derived from adipose tissue (78\%) was significantly higher than that observed in fibroblasts $(0.8 \%)$ and stem cells derived from Wharton's jelly $(0.1 \%)$, placental tissue (0.4\%), and bone marrow (6\%).

\section{CD105}

Figure 6 shows the percentage of CD105-positive stem cells derived from Wharton's jelly, adipose tissue, bone marrow, placental tissue, and fibroblasts derived from foreskin. Significant differences in the expression of CD105 between these cells were observed ( $p<0.05$; ANOVA). Tukey's pairwise comparisons revealed that the expression of CD105 in fibroblasts (43\%) was significantly lower than that in stem cells derived from adipose tissue (95\%), Wharton's jelly (96\%), bone marrow (99\%), and placental tissue (99\%). 
I
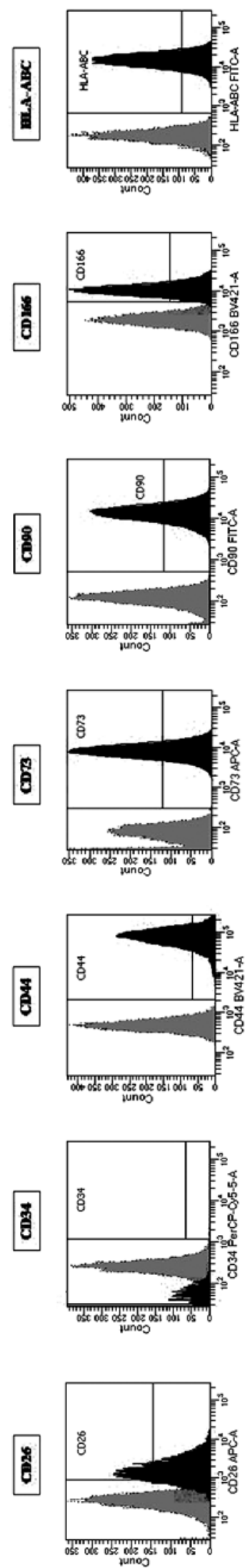

s.JSW-Wg
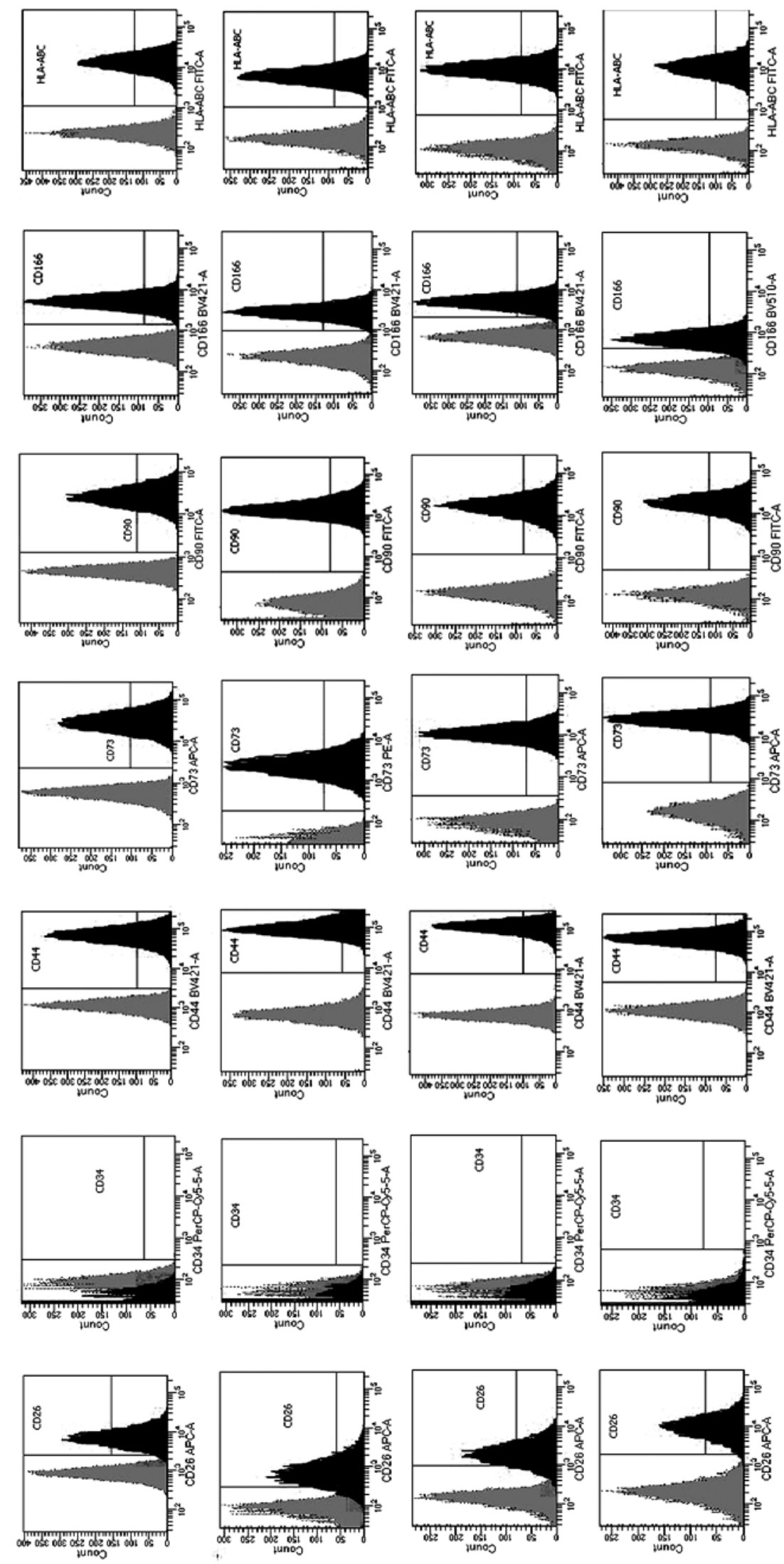

s.JW-rM

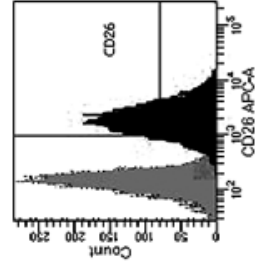

s.SW-Td

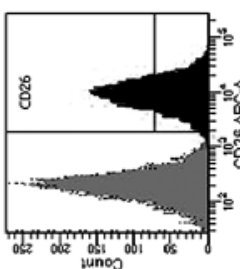

sıse|q0גq!d

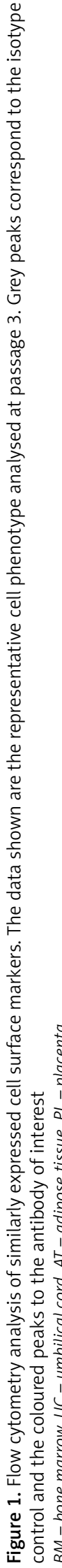



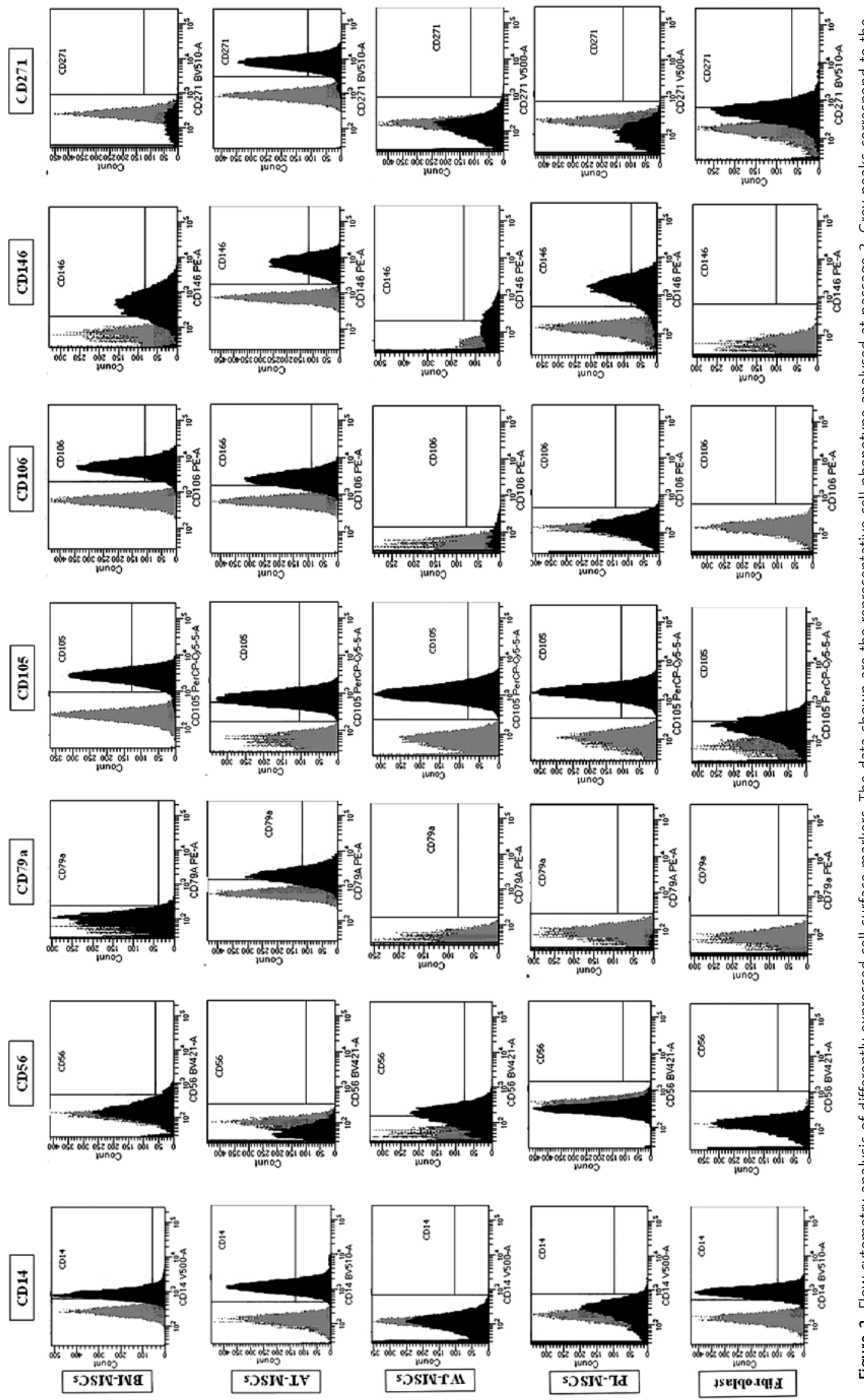

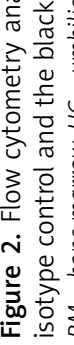




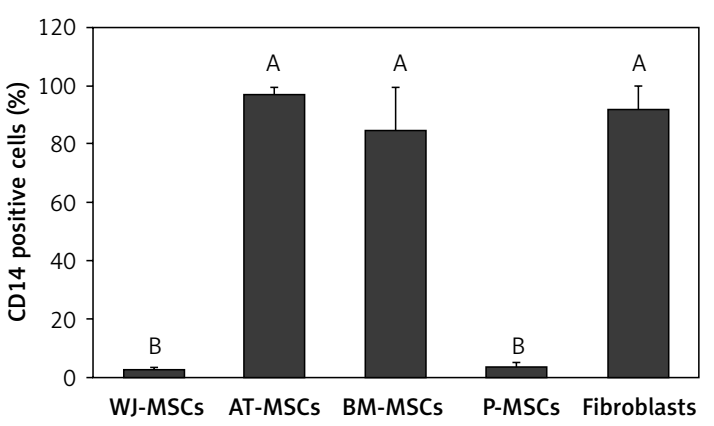

Figure 3. Percentage of CD14-positive cells in stem cells of different origin and fibroblasts derived from foreskin. Data represents means +SD. Means that do not share a letter are significantly different (Tukey's HSD, $p<0.05$ )

MSCs - mesenchymal stem cells, WJ - Wharton's jelly, $A T$ - adipose tissue, $B M$ - bone marrow, $P$ - placental tissue.

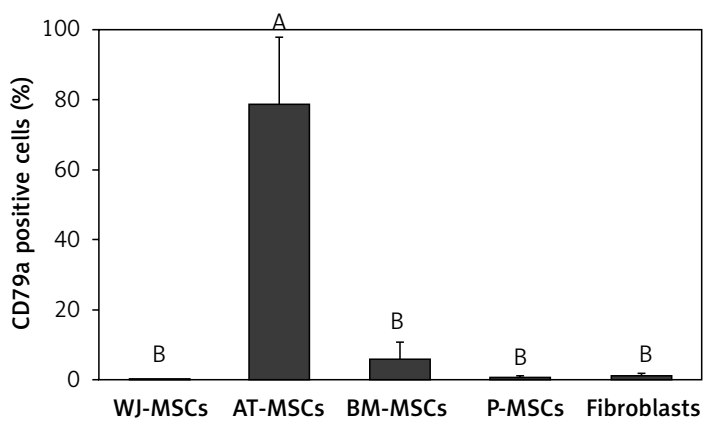

Figure 5. Percentage of CD79a-positive cells in stem cells of different origin and fibroblasts derived from foreskin. Data represents means +SD. Means that do not share a letter are significantly different (Tukey's HSD, $p<0.05$ )

MSCs - mesenchymal stem cells, WJ - Wharton's jelly, $A T$ - adipose tissue, BM - bone marrow, $P$ - placental tissue.

\section{CD106}

The percentage of CD106-positive stem cells (Figure 7) was found to vary in fibroblasts and stem cells from different origins ( $p<0.05$; ANOVA). Tukey's pairwise comparison revealed that the expression of CD106 was significantly lower in fibroblasts (0.4\%) and in stem cells derived from Wharton's jelly (2\%) and placental tissue (10\%) than in stem cells derived from bone marrow (79\%) and adipose tissue (88\%).

\section{CD146}

Figure 8 shows the percentage of CD146-positive stem cells from different sources, as well as fibroblasts. One-way ANOVA revealed that the differences in the levels of expression of CD146 in these cells were statistically significant $(p<0.05)$. Tukey's pairwise comparison revealed that expression of CD146 was significantly lower in fibroblasts $(0.1 \%)$ than stem

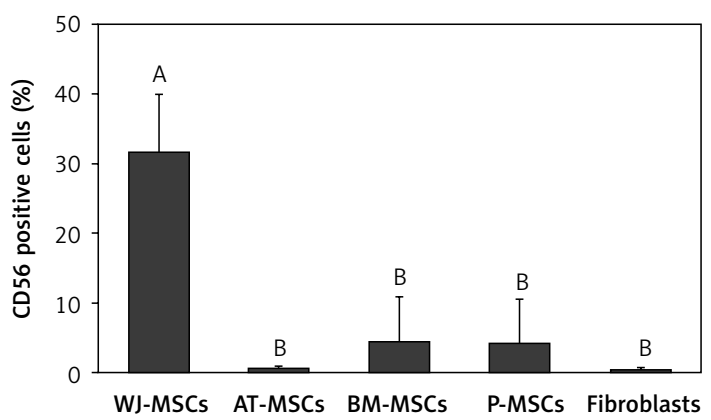

Figure 4. Percentage of CD56-positive cells in stem cells of different origin and fibroblasts derived from foreskin. Data represents means $+S D$. Means that do not share a letter are significantly different (Tukey's HSD, $p<0.05$ )

MSCs - mesenchymal stem cells, WJ - Wharton's jelly, AT - adipose tissue, BM - bone marrow, $P$ - placental tissue.

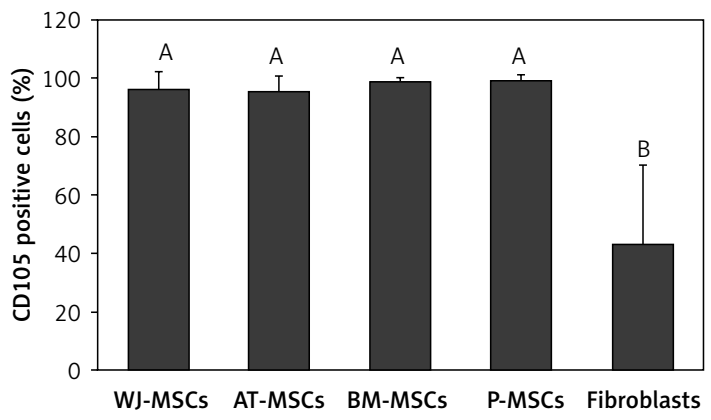

Figure 6. Percentage of CD105-positive cells in stem cells of different origin and fibroblasts derived from foreskin. Data represents means +SD. Means that do not share a letter are significantly different (Tukey's HSD, $p<0.05$ )

MSCs - mesenchymal stem cells, WJ - Wharton's jelly, $A T$ - adipose tissue, BM - bone marrow, $P$ - placental tissue.

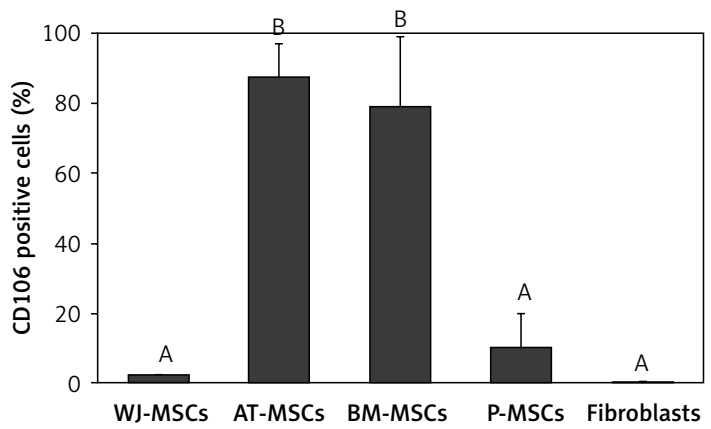

Figure 7. Percentage of CD106-positive cells in stem cells of different origin and fibroblasts derived from foreskin. Data represents means +SD. Means that do not share a letter are significantly different (Tukey's HSD, $p<0.05$ )

MSCs - mesenchymal stem cells, WJ - Wharton's jelly, $A T$ - adipose tissue, BM - bone marrow, $P$ - placental tissue

cells derived from Wharton's jelly (34\%), bone marrow (80\%), placental tissue (85\%), and adipose tissue (96\%). 


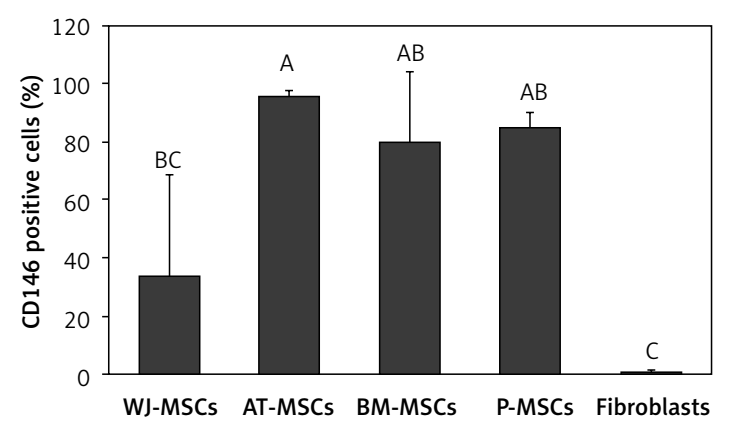

Figure 8. Percentage of CD146-positive cells in stem cells of different origin and fibroblasts derived from foreskin. Data represents means +SD. Means that do not share a letter are significantly different (Tukey's HSD, $p<0.05$ )

MSCs - mesenchymal stem cells, WJ - Wharton's jelly, $A T$ - adipose tissue, $B M$ - bone marrow, $P$ - placental tissue.

\section{CD271}

Figure 9 shows the percentage of CD271-positive stem cells derived from Wharton's jelly, adipose tissue, bone marrow, placental tissue, and fibroblasts derived from foreskin. Significant differences in expression of CD271 between these cells were observed ( $p<0.05$; ANOVA). Tukey's pairwise comparison revealed that the expression of CD271 in stem cells derived adipose tissue (98\%) was significantly greater than in cells derived from placental tissue $(0.3 \%)$, bone marrow $(2 \%)$, and Wharton's jelly (3.1\%), as well as fibroblasts (10\%).

\section{Discussion}

In the current study, we collected a panel of surface markers that were duly studied, with varying and sometimes contradicting results. We studied the expression of these markers on MSCs derived from bone marrow, adipose tissue, Wharton's jelly, and placental tissue, as well as fibroblasts isolated from foreskin. It is of utmost importance to establish cell surface markers that will enable definitive discrimination between MSCs and fibroblasts. Not only would this be important in the authentication of cell lines isolated from various tissues, but also would help to improve the yield and differentiation potential of MSC cultures and to prevent the complications that may arise when MSCs are being cultured for clinical applications. Such complications include the possibility of fibroblasts becoming tumourigenic, which may lead to tumour formation after MSC transplantation [18].

The panel used for the multiplex flow cytometry analysis was designed based on the cell surface markers used to authenticate the identity of MSCs by the International Society for Cellular Therapy (ISCT), i.e. that MSCs must express CD105, CD73, and CD90, and lack expression of CD45, CD34,

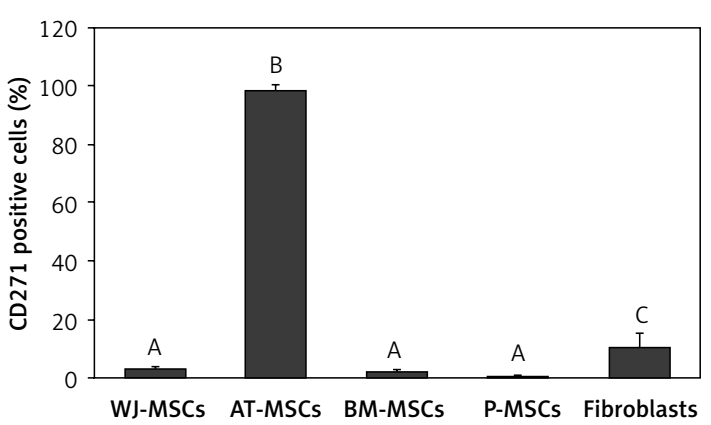

Figure 9. Percentage of CD271-positive cells in stem cells of different origin and fibroblasts derived from foreskin. Data represents means +SD. Means that do not share a letter are significantly different (Tukey's HSD, $p<0.05$ )

MSCs - mesenchymal stem cells, WJ - Wharton's jelly, AT - adipose tissue, BM - bone marrow, $P$ - placental tissue.

CD14 or CD11b, CD79 $\alpha$ or CD19, and HLA-DR [12, 13]. Each of the samples used in the multiplex flow cytometry analysis contained monoclonal antibodies against at least one of each of these positive and negative surface markers, along with several antibodies against the surface markers we aimed to analyse.

Out of the surface markers declared to be positive in MSCs, CD90 was the only one that did not display any significant difference in expression in the MSCs from Wharton's jelly, adipose tissue, bone marrow, and placental tissue, or in fibroblasts from foreskin. This finding comes as no surprise because CD90 plays many roles in cell function including regulation of cell adhesion, apoptosis, and migration [21]. CD105, a surface marker primarily involved in blood vessel development [22], was expressed at similar levels in MSC from all 4 sources (95-99\%), but there was significantly lower expression in fibroblasts (43\%) (Figure 6). Because CD105 has been recognized as a positively expressed marker on all MSCs, this finding could provide a useful insight into the discrimination of fibroblasts from MSCs.

The ISCT have declared several surface markers, including CD34 and CD14, to be negatively expressed on MSCs and fibroblasts [11]. In agreement, we found that CD34 was expressed at low levels $(0.1-1 \%)$ with no significant differences between the different cell types studied. On the other hand, CD14 was expressed at high but similar levels in MSCs derived from adipose tissue and bone marrow and fibroblasts (85-97\%), but at significantly lower levels in MSCs derived from Wharton's jelly and placental tissue (3-4\%) (Figure 3). Therefore, CD14 is a possible discriminating surface marker between fibroblasts and MSCs derived from placental tissue and Wharton's jelly.

CD166/ALCAM (activated leukocyte cell adhesion molecule) is believed to play an important 
role in cellular maintenance and proliferation [23]. It has been recognized as a marker that is positive in both MSCs and fibroblasts [16, 24]. Although unconfirmed, it was believed that CD166 may be useful in distinguishing MSCs from fibroblasts in that the expression of CD166 was lower in MSCS than in fibroblasts. However, we found no significant difference in the expression of CD166 between all the cell types studied (91-100\%).

CD79 $\alpha$ is almost exclusively known to be expressed on B-cells and B-cell neoplasms and has been declared to be negatively expressed on MSCS $[13,25]$. Our findings, however, contradict this in that MSCs derived from adipose tissue displayed a significantly higher expression of CD79 $\alpha$ than MSCs from the other cell types and fibroblasts (Figure 5).

CD271, a member of the tumour necrosis factor receptor superfamily that plays an important role in neuronal cell survival, differentiation, and migration [26], has been suggested as a candidate for use as a single definitive marker to identify and isolate MSCs from all other cell types that are present in the tissue from which they are derived [27]. In contrast, we found that CD271 was expressed at high levels only in MSCs derived from adipose tissue (98\%), whereas the MSCs from the other sources had very low expression of this marker $(0.4-3 \%)$, and this was also true of fibroblasts (10\% expression).

Our findings correlated with those of previous studies regarding the expression of CD44 and HLA-ABC [16, 28-30]. The expression of these 2 surface markers was not found to be significantly different between MSCs and fibroblasts.

CD26, a marker associated with CD4+ T cells that functions in T-cell signal transduction [31], has been claimed in previous studies to be fibroblast-specific [18], while other studies found that the expression of this marker varied among MSCs samples [32], and so there has been no conclusive result as to how the expression of CD26 compares between fibroblasts and MSCs. We found that there was no significant variation in the expression of this marker between fibroblasts and MSCS derived from Wharton's jelly, adipose tissue, bone marrow, or placental tissue (89-99\%).

CD106, a cell surface marker that has been found to mediate cell-cell adhesion and plays a critical role in MSC-mediated immunosuppression [33-35], is another marker that has been suggested to be useful in the discrimination between fibroblasts and MSCs; it has been declared by some to be MSC-specific and by others to be expressed in MSCs at a significantly higher level than in fibroblasts $[16,18]$. Our findings supported this claim when comparing the expression of CD106 in fibroblasts with MSCs derived from adi- pose tissue and bone marrow. Indeed, MSCs from these 2 sources had significantly higher expression $(79-88 \%)$ than fibroblasts (0.4\%). However, CD106 would not be a reliable marker for the discrimination between fibroblasts and MSCs derived from placental tissue and Wharton's jelly due to the absence of significant differences in CD106 expression between these cell types (Figure 7).

It has been concluded that CD146 is MSC specific [16], and our findings support this conclusion. We found that fibroblasts did not express CD146, while MSCs from different sources all expressed this marker at varying levels. We therefore conclude that CD146 can be used as a marker to distinguish MSCs from fibroblasts (Figure 8).

The expression of CD56 has also displayed nonuniform results in several studies [25, 26], and there has, therefore, been no definitive conclusion as to whether or not it is of use in the discrimination between MSCS and fibroblasts. In the current study, the expression of CD56 was only significantly higher in MSCs derived from Wharton's jelly than in fibroblasts, as well as MSCs derived from adipose tissue, bone marrow, and placental tissue (Figure 4). We can therefore conclude that CD56 would not be useful in the discrimination between MSCs and fibroblasts.

In conclusion, the results of our study indicate that the following markers may be used to differentiate between fibroblasts and MSCs derived from the following: adipose tissue - CD79a, CD105, CD106, CD146, and CD271; Wharton's jelly - CD56, CD14, and CD105; bone marrow CD105, CD106, and CD146; and placental tissue - CD14, CD105, and CD146. The results of the current study warrant further investigations to confirm the differences in surface marker expression between MSCs and fibroblasts using RT-PCR and/ or immunohistochemistry.

\section{Acknowledgments}

This work was supported by the Deanship of Research at Jordan University of Science and Technology (Grant Number: 20160245).

\section{Conflict of interest}

The authors declare no conflict of interest.

\section{References}

1. Kumar V, Abbas AK, Aster JC. Robbins Basic Pathology. 10th ed. Elsevier Saunders, Philadelphia 2018.

2. Riordan NH, Morales I, Fernández G, et al. Clinical feasibility of umbilical cord tissue-derived mesenchymal stem cells in the treatment of multiple sclerosis. J Transl Med 2018; 16: 57.

3. Blumberg SN, Berger A, Hwang L, Pastar I, Warren SM, Chen $\mathrm{W}$. The role of stem cells in the treatment of diabetic foot ulcers. Diabetes Res Clin Pract 2012; 96: 1-9. 
4. Stuckey DW, Shah K. Stem cell-based therapies for cancer treatment: separating hope from hype. Nat Rev Cancer 2014; 14: 683-91.

5. De Bari C, Roelofs AJ. Stem cell-based therapeutic strategies for cartilage defects and osteoarthritis. Curr Opin Pharmacol 2018; 40: 74-80.

6. Iyer NR, Wilems TS, Sakiyama-Elbert SE. Stem cells for spinal cord injury: strategies to inform differentiation and transplantation. Biotechnol Bioeng 2017; 114: 245-59.

7. Antebi B, Mohammadipoor A, Batchinsky Al, Cancio LC. The promise of mesenchymal stem cell therapy for acute respiratory distress syndrome. J Trauma Acute Care Surg 2018; 84: 183-91.

8. Litwinowicz R, Kapelak B, Sadowski J, Kędziora A, Bartus $K$. The use of stem cells in ischemic heart disease treatment. Kardiochir Torakochir Pol 2018; 15: 196-9.

9. Yan X, Cheng X, He X, Zheng W, Yuan X, Chen H. Hypothermia enhances heme oxygenase 1-expressing mesenchymal stem cell-induced repair of cerebral hypoxia-ischemia. Arch Med Sci 2019; doi: 10.5114/ aoms.2019.86784.

10. Mafi R, Hindocha S, Mafi P, Griffin M, Khan W. Sources of adult mesenchymal stem cells applicable for muscu loskeletal applications - a systematic review of the literature. Open Orthop J 2011; 5: 242-8.

11. Semon JA, Maness C, Zhang X, Sharkey SA, Beuttler MM, Shah FS. Comparison of human adult stem cells from adipose tissue and bone marrow in the treatment of experimental autoimmune encephalomyelitis. Stem Cell Res Ther 2014; doi: 10.1186/scrt391.

12. Horwitz EM, Le Blanc K, Dominici M, Mueller I, Slaper-Cortenbach I, Marini FC. Clarification of the nomenclature for MSC: The International Society for Cellular Therapy position statement. Cytotherapy 2005; 7: 393-5.

13. Dominici M, Le Blanc K, Mueller I, Slaper-Cortenbach I, Marini F, Krause D. Minimal criteria for defining multipotent mesenchymal stromal cells. The International Society for Cellular Therapy position statement. Cytotherapy 2006; 8: 315-7.

14. Le Blanc K, Davies LC. MSCs - cells with many sides. Cytotherapy 2018; 20: 273-8.

15. Lin CS, Xin ZC, Dai J, Lue TF. Commonly used mesenchymal stem cell markers and tracking labels: limitations and challenges. Histol Histopathol 2013; 28: 1109-16.

16. Halfon S, Abramov N, Grinblat B, Ginis I. Markers distinguishing mesenchymal stem cells from fibroblasts are downregulated with passaging. Stem Cells Dev 2010; 20: 53-66.

17. Ichim TE, O'Heeron, P, Kesari S. Fibroblasts as a practical alternative to mesenchymal stem cells. J Transl Med 2018; 16: 212

18. Kundrotas $\mathrm{G}$. Surface markers distinguishing mesenchymal stem cells from fibroblasts. Acta Med Litu 2012; 19: 75-9.

19. Watson JT, Foo T, Wu J, et al. CD271 as a marker for mesenchymal stem cells in bone marrow versus umbilical cord blood. Cells Tissues Organs 2013; 197: 496-504.

20. Abu-Ameerh MA, Jafar HD, Hasan MH, et al. Platelet lysate promotes re-epithelialization of persistent epithelial defects: a pilot study. Int Ophthalmol 2019; 39: 1483-90.

21. Kumar A, Bhanja A, Bhattacharyya J, Jaganathan BG. Multiple roles of CD90 in cancer. Tumour Biol 2016; 37: 11611-22.

22. Fonsatti E, Sigalotti L, Arslan P, Altomonte M, Maio M. Emerging role of endoglin (CD105) as a marker of angiogenesis with clinical potential in human malignancies. Curr Cancer Drug Targets 2003; 6: 427-32.
23. Smith NR, Davies PS, Levin TG, et al. Cell adhesion molecule CD166/ALCAM functions within the crypt to orchestrate murine intestinal stem cell homeostasis. Cell Mol Gastroenterol Hepatol 2017; 3: 389-409.

24. Maleki M, Ghanbarvand F, Reza Behvarz M, Ejtemaei M, Ghadirkhomi E. Comparison of mesenchymal stem cell markers in multiple human adult stem cells. Int J Stem Cells 2014; 7: 118-26.

25. Kwon A, Kim Y, Kim M, et al. Tissue-specific differentiation potency of mesenchymal stromal cells from perinatal tissues. Sci Rep 2016; 6: 23544.

26. Murillo-Sauca O, Chung MK, Shin JH, et al. CD271 is a functional and targetable marker of tumor-initiating cells in head and neck squamous cell carcinoma. Oncotarget 2014; 5: 6854-66.

27. Flores-Torales E, Orozco-Barocio A, Gonzalez-Ramella OR, Carrasco-Yalan A, Gazarian K, Cuneo-Pareto S. The CD271 expression could be alone for establisher phenotypic marker in Bone Marrow derived mesenchymal stem cells. Folia Histochem Cytobiol 2010; 48: 682-6.

28. Lorenz K, Sicker M, Schmelzer E, et al. Multilineage differentiation potential of human dermal skin derived fibroblasts. Exp Dermatol 2008; 17: 925-32.

29. Alt E, Yan Y, Gehmert S, Song YH, Altman A, Gehmert S, Bai X. Fibroblasts share mesenchymal phenotypes with stem cells, but lack their differentiation and colony forming potential. Biol Cell 2011; 103: 197-208.

30. Fu X, Chen Y, Xie FN, et al. Comparison of Immunological Characteristics of mesenchymal stem cells derived from human embryonic stem cells and bone marrow. Tissue Eng Part A 2015; 21: 616-26.

31. Hatano R, Ohnuma K, Yamamoto J, Dang NH, Morimoto C. CD26-mediated co-stimulation in human CD8(+) $T$ cells provokes effector function via pro-inflammatory cytokine production. Immunology 2013; 138: 165-72.

32. Cappellesso-Fleury S, Puissant-Lubrano B, Apoil PA, et al. Human fibroblasts share immunosuppressive properties with bone marrow mesenchymal stem cells. J Clin Immunol 2010; 30: 607-19.

33. Yang ZX, Han ZB, Ji YR, et al. CD106 identifies a subpopulation of mesenchymal stem cells with unique immunomodulatory properties. PLoS One 2013; 8: e59354. doi: 10.1371/journal.pone.0059354.

34. Battula VL, Treml S, Bareiss PM, et al. Isolation of functionally distinct mesenchymal stem cell subsets using antibodies against CD56, CD271, and mesenchymal stem cell antigen-1. Haematologica 2009; 9: 173-84.

35. Khan WS, Adesida AB, Tew SR, Lowe ET, Hardingham TE. Bone marrow-derived mesenchymal stem cells express the pericyte marker $3 \mathrm{G} 5$ in culture and show enhanced chondrogenesis in hypoxic conditions. J Orthop Res 2010; 28: 834-40. 